\title{
Reform of health-care payments for Chinese medical insurancepractices in Luohu District, Shenzhen City, Guangdong Province
}

\author{
Fangfang Gong \\ Luohu Hospital Group \\ Xizhuo Sun ( $\square$ xizhuomd@163.com ) \\ Wenhai Li \\ Luohu Hospital Group \\ Zou Zhang \\ Beijing Normal University - Zhuhai Campus \\ Yanan Li \\ Luohu Hospital Group
}

Research article

Keywords: Medical Insurance System, Health-oriented, Reform

Posted Date: October 8th, 2019

DOI: https://doi.org/10.21203/rs.2.15711/v1

License: (1) (i) This work is licensed under a Creative Commons Attribution 4.0 International License.

Read Full License 


\section{Abstract}

Background Following the implementation of the Healthy China 2030 strategy, China's health-care system must shift from being disease-centered to health-centered. Medical insurance funds are the main economic resource for medical health-care service providers in China; therefore, the Chinese medical insurance system has become an important economic lever for adjusting the behavior of medical healthcare providers. In the new round of medical reform, substantial progress has been made in the construction of a medical treatment insurance system. The world's largest medical insurance network has been created in a relatively short period in China and basically achieves universal medical insurance coverage. However, this system mainly provides full coverage to the amount and has yet to fully achieve the principle of "health-care for all" proposed by the Healthy China 2020 strategy. China must promote reform in the medical insurance system and establish a medical insurance guidance mechanism to ensure that medical service providers consider and promote health care.

Methods Using Luohu Hospital Group in Shenzhen City, Guangdong Province as the research object, the details of the health maintenance organization's reform of its medical insurance payment patterns to be more health-oriented are introduced. Comparing the summarized characteristics of the health maintenance organization's payment patterns, the relevant data for the medical insurance operation and health status of the insured before and after the reform were analyzed statistically.

Results The data show that after the reform, the total hospitalization cost of the insured, number of inpatients, and hospitalization rate all decreased. The growth rate of expenditure in the medical insurance fund slowed and initial results were shown in preventive health-care work. The incidence of some infectious diseases and the hospitalization rate of patients with chronic diseases decreased.

Conclusions The medical service providers form positive incentives and appropriate medical orientations, while patients demanding health care may form good habits of seeking medical treatment and healthy life, but not pursuing economic benefits through the medical insurance reform.

\section{Background}

In March 2015, the concept of "Healthy China 2030" was raised during the "Two Sessions" (the general term for the meeting of the National People's Congress of the People's Republic of China and the Chinese People's Political Consultative Conference in China's highest decision-making hall) and it was entered into the government's work report. The Healthy China 2030 framework explicitly proposed to "promote the construction of healthy China, deepen the reform of medical and health system, and practice medical service, medical insurance and medicine coordination."In August 2016, Xi Jinping, the General Secretary of the Central Committee of the Communist Party of China, stressed the strategic position of prioritizing people's health at the first Chinese National Health and Fitness Conference, which marked a new chapter in the construction of a healthy China. Against this background, the basic national Chinese health insurance system, i.e., medical insurance, should play an important role in promoting a healthy China. 
The basis for undertaking this heavy responsibility comes from the strong incentives and restraining effect of medical insurance on medical services' behavior. Differing medical insurance payment methods will lead to completely different medical behaviors and they can also guide medical workers to consider and promote the health of the insured over the uninsured. The core of Healthy China 2030 is to promote and improve health conditions. Healthy China 2030 gives medical insurance economic leverage over health-care services to guide medical service providers to shift from providing "disease-centered" to "health-centered" services and strive to achieve universal health-care coverage. Therefore, the exploration and establishment of Chinese health-oriented payment patterns in medical insurance should be an important link in the construction of a healthy China by 2030 .

Establishing universal medical insurance and expanding basic medical insurance coverage.The construction of a universal medical insurance system for China started in 1998 and continued until 2002. In 1998, the State Council issued a "Decision to Establish a Basic Medical Insurance System for Urban Workers" (GF [1998] No. 44), which formally established a medical insurance system for urban workers in China and marked the beginning of the comprehensive development of a medical insurance system for urban workers in China. Since 2003, universal medical insurance has been developing rapidly. In 2003, the pilot work for the New Cooperative Medical Care (NCMS) system was launched nationwide. NCMS's goal for full coverage was achieved 2 years ahead of schedule at the end of June 2008. In 2007, the State Council issued the "Guidance on Carrying out Pilot Programs of Basic Medical Insurance for Urban Residents" (GF [2007] No. 20), which marked the official launch of a medical insurance system for urban residents. Thus, a medical insurance system with universal health coverage was basically formed. ${ }^{1}$ In 2009, a landmark document launched new medical reform. The "Opinions on Deepening the Reform of Medical and Health System" pointed out that "China's basic medical security system consists of medical insurance for urban workers, medical insurance for non-working urban residents and NCMS, covering the urban working population, non-working urban population and rural population respectively." In 2010, the Standing Committee of the National People's Congress promulgated the Social Insurance Law (Fig. 1.), which forced the legal development of a basic medical insurance system. After years of efforts, China established a medical security network covering $96.5 \%$ of the country's population through the provision of the NCMS and medical insurance systems for urban workers and unemployed urban residents.

The increasingly serious expenditure pressure on medical insurance funds.Employee medical insurance funds have been operating for a long time and provide a good example of expenditure pressures. The growth of the national economy will slow after it enters a new developmental stage. Accordingly, employees' salary growth will also slow; therefore, the income growth of medical insurance funds charged in proportion to the employees' salaries will also decrease. However, under the influence of rising medical costs, increasing demand for medical services, acceleration of population aging, increase in the number of patients with chronic diseases, and other factors, medical insurance fund expenditures still maintain high growth. From 2009 to 2018, the average growth rate of employee medical insurance fund expenditure was $16.63 \%$, which is higher than the fund income's average growth rate of $16.24 \%$ (Fig. 2). Many Chinese regions had deficits in their medical insurance funds. More seriously, the growth rate of 
medical insurance funds' per capita expenditure (11.97\%) was higher than the growth rate of China's per capita gross domestic product $(2.89 \%)$ and the growth rate of per capita disposable income $(9.04 \%)$. If we cannot continue to strengthen control over health-care expenditures, it will be difficult for the medical insurance system to achieve sustainable development at the current growth rate.

Income

Income

Expenditure

Expenditure

The heavy economic burden for patients to seek medical treatment.From 2009 to 2018, China's total health expenditure sustained rapid growth. The national total health expenditure increased from 1.8 trillion yuan in 2009 to 5.8 trillion yuan in 2018 , with an average annual growth of $14.2 \%$, and the total health expenditure per capita increased from 1,314 yuan to 4,148 yuan. ${ }^{2}$ Sources of health costs mainly include government, social, and personal health-care expenditure (Table 1). Following the development of China's medical insurance system, the proportion of personal health-care expenditure fell annually from $37.46 \%$ in 2009 to $28.7 \%$ in 2018 . However, this decline has slowed significantly since 2015 . There is still a large gap between this value and the World Health Organization's (WHO) proposed $10 \%-15 \%$ fair financing system; therefore, patients still experience a relatively heavy financial burden for their medical treatment. At a national conference on health poverty alleviation in 2018, Liu Kui, Deputy Director of the Financial Department at the National Health Commission, pointed out that the proportion of diseasecaused poverty among the poor households recorded in China were both above $42 \%{ }^{3}$ This has become a major problem in China, which needs to eliminate poverty in building a healthy society.

\section{Methods}

China's medical insurance system currently has basically realized a full-coverage system for its population. However, there is still a long way to go before China realizes the WHO's global strategy for achieving universal health care for all. That is, China, must adopt primary health-care strategies, with institutions, systems, and mechanisms as the guarantee, to provide the Chinese public with fair, comprehensive, and effective basic medical health-care services at reduced costs, and finally achieve the purpose of improving public health. ${ }^{4}$ To realize the qualitative change from universal coverage to universal health, China's current health-care services concept and model centered on treatment of diseases must be reversed to become health-centered. This change requires a comprehensive combination of administrative, economic, and legal means using medical insurance as economic leverage to undertake this important task. The function of medical insurance in guiding the behavior of medical services is mainly realized through their payment of medical expenses to the medical service providers. After more than 20 years in China, great achievements have been made in medical insurance payment patterns, which play an important role in guiding medical workers to properly treat patients while 
simultaneously controlling their medical expenses. In looking at the wider perspective of a healthy China, we always consider whether these payment methods fairly guide medical workers and promote the health of the insured. The answer is clearly disappointing. An early implementation of the payment-by-item principle led to medical insurance organizations compensating for expenses compensation according to the amount of medical services provided by medical institutions. This encouraged medical institutions to provide more services and increase the amount of treatment required. After gradually introducing a variety of fixed prepayments, i.e., medical insurance organizations set the maximum compensation from their clients' medical treatment insurance for each hospitalization. Although the service items provided in single medical treatments at medical institutions decreased, the number of patient hospitalizations increased significantly. In response to this situation "separated hospitalization" emerged in medical institutions; i.e., before the hospitalized patients have fully recovered, they are required to be discharged and go through hospitalization procedures multiple times to avoid the maximum limit of their medical insurance. This reduces these patients' satisfaction with their medical treatment and increases its difficulty and cost. According to data released by the National Health Commission, Chinese residents' rate of hospitalization had increased from $9.9 \%$ in 2009 to $17.6 \%$ in 2017 . The disease type scores and diagnosis-related groups introduced in recent years are now upgrading diagnoses to include more patients. The capitation payment for outpatient coordination, which could guide doctors to consider health was implemented in relatively few areas. For example, outpatient coordination has been implemented for nearly a decade in Guangdong Province, but capitation payment is only implemented in Shenzhen, Zhuhai, and Dongguan. However, the effect is reduced because big hospitals compete for primary patients and primary medical resources.

Reform of health-oriented medical insurance payment patterns in practice in Luohu District, Shenzhen. The essence of the reform of health-oriented medical insurance payment patterns is to guide medical service providers to consider and promote health care for their patients. What kind of medical insurance payment methods can guide medical providers to consider their patients' health care? Theoretically, arrangements that link a doctor's income to an insured person's health should be considered as a healthoriented payment method. In reviewing medical insurance system reform worldwide, two major models can meet the above requirements: i.e., the gatekeeper system of family doctors in Britain, Canada, and other countries, and the American management-centered medical care system, including health maintenance organizations (HMO) and patient-centered medical homes that emerged later. Marcial et al. observed that the British gatekeeper system forces patients to refer to their family doctor before they can have their first medical treatment in primary medical institutions, with general practitioners providing the primary health services. In terms of medical insurance payment patterns, the combination of total budget and treatment payments was adopted to guide general practitioners to retain more medical insurance funds by improving their residents' health condition and providing more preventive health-care and management services for residents, which achieved a win-win situation to some extent. ${ }^{5}$ Flood et al. noted that the membership of American HMOs pay fixed medical costs and the HMO provides all their medical services. ${ }^{6}$ To ensure that the cost of received medical services does not exceed the cost during the service 
period, the HMOs undertake various efforts to improve the health status of the served population, thereby reducing their overall morbidity and possible medical expenses.

In learning from the international literature on using medical insurance payment patterns to promote health in combination with the specific characteristics of the Chinese medical insurance system, we believe that when the health-care and medical responsibilities of a group of insured people are contracted to a medical service provider together with their medical insurance costs (i.e., the healthier the insured are, the better the medical provider's economic benefits), then an economic mechanism is established to guide these doctors to actively consider the health of the insured. Considering this principle, the Shenzhen Municipal Ministry of Human Resources and Social Security Bureau, Shenzhen Municipal Health Commission, and the Luohu District Government jointly issued the "Implementation Plan of Medical Insurance Total Amount Management System Integrating Pilot Establishment and Hierarchical Medical Treatment of Shenzhen" in 2016. The Luohu Hospital Group was the pilot institution to test the reform of medical insurance payment patterns, i.e., "total amount management, balance for reward," with social medical insurance enrollees who signed a family doctor service agreement as the pilot group. Under the premise of not forcing changes in the medical treatment behavior of the insured, the medical health-care responsibility of the above-mentioned insured were contracted to Luohu Hospital Group together with their medical insurance expenses. If there was a balance in liquidation at the end of the year, the hospital group will receive the remaining balance for the continued performance of preventive health care and improving the treatment of medical workers. ${ }^{7}$ The main calculation methods are as follows:

Total amount of medical insurance management in 2017 = total amount of medical insurance actually incurred by the contracted insured in 2016 * $(1+$ average growth rate of medical insurance fund expenditure in Shenzhen in 2017)

The eligible contracted insured refer to those who successively participated in Shenzhen social medical insurance for more than 2 years and signed a family doctor service agreement with Luohu Hospital Group as of December 31, 2017. Thus, the total amount of medical insurance management is a dynamic data point that is constantly adjusted with changes in the number of insured.

Balance in 2017 = the total amount of medical insurance occurred in 2017-the total amount of medical insurance management in 2017

Characteristics of the reform of medical insurance payment patterns in Luohu District. Following the reform of medical insurance payment patterns in Luohu District, in the international and domestic exchange of experiences, some experts proposed that "the payment pattern reform of medical insurance in Luohu is HMO, and it is a complete copy of HMO." The two systems do have something in common, which is the basic operational principle, i.e., "total amount management, balance for reward." To obtain the surplus rewards, both systems attach importance to preventive health care for their residents. However, after a specific comparative analysis, we found that there are many differences between the 
two. These differences are the innovation and characteristics of Luohu's medical insurance payment reform.

Residents' right to seek medical treatment.The members of HMOs have a very limited choice of medical treatment. That is, $\mathrm{HMO}$ members can only seek medical treatment in $\mathrm{HMO}$-affiliated institutions insuring them and medical treatment expenses outside the HMO system cannot be reimbursed. In contrast, the reform of Luohu District is based on the premise that the right of the insured to seek medical treatment is not restricted. The insured can go to any medical institution for medical treatment according to the needs of his/her disease without the approval of Luohu Hospital Group and the incurred medical expenses will be reimbursed, which guarantees the residents' right to choose medical treatment to the greatest extent.

Risk selection and service content.In HMO medical services, insurance organizations can reduce the underwriting risks through adverse selection. Residents must be audited before signing a contract with an HMO. Only those who pass the insurance organization's audit can purchase different levels of insurance from the HMO. As members of the HMO, they can enjoy medical care services corresponding to the level of insurance purchased. However, this leads to "cream skimming," i.e., insurance organizations only accept residents with relatively better health conditions for insurance coverage. Those with worse health conditions are declined politely, which will seriously harm the patients' interests. In terms of health-care service contents, HMO members can only enjoy the medical services stipulated in their agreement. The latitude for doctors to practice medicine is restricted under HMOs, and influenced by the concept of fee control, these doctors are likely to reduce necessary prescriptions and examinations to cut down costs, which seriously affects the quality of medical services for their patients. The adverse selection by HMOs and their limited medical services are important reasons for the decline of this service model in the 21st century. In contrast, the reform of Luohu District designed a one-way selection mechanism, i.e., Luohu residents can choose whether to sign a contract or not, and medical institutions are not allowed to refuse residents. The reform plan clearly stipulates that the "Luohu Hospital Group shall not refuse eligible insured to participate in the pilot program for any reason," which effectively avoids the improper risk selection by medical institutions and guarantees the residents' right to choose the type of health care they wish to receive. In terms of service content, the reform plan clearly indicates that in addition to signing the registration agreement to provide basic medical and public health-care services, Luohu Hospital Group also needs to provide family doctor services, a dynamic electronic health record management service, chronic disease management services, medication consultation and guidance services, preventive healthcare services, family sickbed services, community rehabilitation services, and medical integration pension services among other personalized services.

Medical insurance payment patterns provide different guidance for doctors. The outside medical treatment expenses of HMO members cannot be reimbursed; therefore, the HMO is likely to make excuses to not accept patients so that they can reduce their expenditures and obtain more funds under the "balance for reward" mechanism, especially for patients with difficult and severe diseases who really need medical services. In Luohu District's reform plan for medical insurance payment patterns, the medical insurance expenses of the insured who go for medical treatment outside Luohu Hospital Group 
are clearly stipulated to be drawn from the hospital group's total amount. That is, if Luohu Hospital Group cannot attract its patients to stay in the group through providing effective services, their patients can freely go to other medical institutions to receive medical treatment. Luohu Hospital Group has no right to interfere in the management of medical insurance expenses for patients receiving health care outside the group. Because of this design, Luohu Hospital Group does not dare to shuffle patients and reduce the quality of its services. Thus, the uniqueness of the "balance for reward" approach is assured. The residents' health is at the core of Luohu Hospital Group's health-care services, i.e., good preventive health care means that the residents get sick less and are hospitalized fewer times. This frees up resources for improving service quality and contents to attract patients to the Luohu Hospital Group, thus realizing the qualitative change of insurance from "disease insurance" to "health insurance."

\section{Results}

In 2018 , a total of 520,000 residents signed a family doctor service agreement with Luohu Hospital Group. About 380,000 of these residents met the requirements of the pilot program (i.e., patients who drew social medical insurance from Shenzhen, who are living or working in Luohu District and have been insured for 2 years or more). The analysis of the effect of the reform was based on these 380,000 residents.

The basic data, such as medical expenses, medical insurance fund expenditure, and the number of hospitalizations of the contracted insured were respectively collected from medical insurance organizations in Shenzhen and Luohu District for statistical analysis. The data were sorted according to the number of hospitalizations and medical expenses for different disease types. In combination with Luohu Hospital Group's preventive health-care measures, the reasons for the changes in the rankings and the implementation effects of the preventive health-care measures were analyzed. Get data on the effectiveness of preventive health care in the area from the Centers for Disease Control, and extract relevant data from the Luohu Hospital Group according to the disease type.

Decreased total cost of hospitalization for the contracted insured. The total cost of hospitalization for the contracted insured increased rapidly at the beginning of the reform. The annual growth rates were $43.52 \%$ and $25.77 \%$ in 2016 and 2017, respectively. However, they began to decrease in 2018, with a decrease of $0.73 \%$ compared with that in 2017 (Fig. 3).

\section{Discussion}

\section{The growth rate of medical insurance fund expenditure is lower than the average level for Shenzhen}

The growth rate of medical insurance funds in Shenzhen City is measured by the growth rate of medical insurance fund expenditure per capita. From 2016 to 2018, the per capita medical insurance fund of Luohu Hospital Group's contracted insured increased annually, with an increase from 1,477 yuan to 1,689 


\section{The average cost of hospitalization of the contracted insured in Luohu Hospital Group is significantly lower than the level outside the group}

From 2016 to 2018, compared with the average cost of hospitalization, the expenditure on medical expenses in the Luohu Hospital Group was lower than that outside the group, while the average cost of hospitalization outside the group was $35 \%$ to $46 \%$ higher than that in the group (Fig. 4).

\section{Decreased number of hospitalizations for the contracted insured}

At the beginning of the reform, the number of hospitalizations for the contracted insured increased rapidly. The annual growth rates were $32 \%$ and $20.4 \%$ in 2016 and 2017, respectively. However, the number of hospitalizations began to decrease in 2018 , with a decrease of $14.7 \%$ compared with that in 2017 during the same term (Fig. 5).

2018 year

2018 year

\section{Analysis of the top five disease types with the number of hospitalizations of the contracted insured}

According to rankings of the number of hospitalizations and disease types of the contracted insured (Table 3), the rankings of the top five disease types from 2016 to 2018 did not change greatly, but a slight change was observed compared with the number of onset cases. This study analyzes the changes in rankings of disease types and number of hospitalizations according to the medical reform measures in Luohu District, i.e., mainly the preventive health-care measures.

First, the number of hospitalizations due to diseases like pulmonary infection continued to decrease in 2017 and 2018, decreasing from 2,727 cases in 2016 to 1,966 cases in 2018, with an overall decrease of $27.91 \%$ (Fig. 6). The Luohu Hospital Group launched a flu and pneumonia vaccination program in July 2016. The scope of vaccination objects continued to expand during this period. In the first stage, the patients were all over 60 years old. In the second stage, the patients included patients with diabetes, chronic obstructive pulmonary diseases, and cardiovascular and cerebrovascular diseases. All residents who signed the family doctor service agreement with Luohu Hospital Group can now voluntarily enjoy 
free vaccinations. By the end of 2018, 39,157 people had received free vaccination services. The variation trend of the number of hospitalizations for diseases like pulmonary infections shows that the vaccination prevention measures taken by Luohu Hospital Group have shown an effect to some extent.

Second, the number of hospitalizations for chronic disease treatment began to decease in 2018. In 2018, the number of hospitalizations for type 2 diabetes decreased by $7.3 \%$ (Fig. 7) compared with that in 2017 , and that for hypertension decreased by $5.4 \%$ (Fig. 8).

Third, the number of hospitalizations for senile cataract surgery is continually increasing. Especially in 2016, the first year of the reform, the number increased by $24.59 \%$ compared with that in 2015 (Fig. 9). The number of hospitalizations for postoperative chemotherapy treatment of malignant tumors also increased rapidly after the reform, but began to decrease in 2018 (Fig. 10). The variation trends of the above two diseases are related to the preventive health-care measures taken by Luohu Hospital Group. For example, elderly people considered their poor eye sight to be presbyopia, but after the community health service center has performed physical examinations and conducted health publicity and education for the elderly, the elderly may identify their cataract disease early and successfully receive cataract surgery as treatment. The potential reasonable medical demands brought by the reform rapidly increased after some time, but gradually decline after reaching a peak because the number of undiscovered patients with potential cataract disease was reduced gradually. Similarly, the potential medical demands for malignant tumors increased rapidly through early screening and then stabilized gradually. Therefore, the increases in reasonable medical demands caused by effective preventive health-care measures are time-limited to some extent. When the increase in demand peaks, it will gradually or rapidly fall according to the characteristics of the disease types. The cost of preventive health care is thus much lower than the medical expense after the onset of disease and the residents' quality of life will also be improved significantly.

Fourth, the number of hospitalizations for diseases such as cervicalgia, omalgia, osphyalgia, and skelalgia (i.e., lumbar disc herniation, cervical joint toughness, mixed cervical spondylosis) began to decrease in 2018 (Fig. 11). In terms of the rankings of disease types, however, the number of hospitalizations for these diseases was the highest over 3 years (Table 3 ). Thus, new requirements for Luohu Hospital Group's preventive health-care work were identified. Next, the group's resources in various disciplines, such as rehabilitation, traditional Chinese medicine, and bone injury, will be fully used in the early prevention of these diseases.

\section{Conclusions}

According to the data analysis, the reform of medical insurance payment patterns has been performed over 3 years in Luohu District, but the medical insurance fund expenditure has never decreased rapidly. Nevertheless, we believe that we should continue to make efforts to reform health-oriented medical insurance payment patterns. First, from a system design perspective, Luohu's reform draws lessons from advanced experiences domestically and internationally, and effectively avoids problems, such as adverse 
selection, residents' restricted medical treatment choices, shifting responsibility back to patients, and reduced quality of medical services. Second, many studies have shown that changes in the expenditure

of medical expenses and medical insurance funds are jointly influenced by various factors, ${ }^{8}$ including the release of potential medical demands, the law of disease development, and changes in disease spectrum, as well as national policies, medical treatment prices, and rising price levels. More importantly, the reform of medical insurance payment patterns in Luohu District led medical institutions to change their medical treatment orientation by turning from treatment-centered to health-centered care. Various preventive health-care measures also have good results that improve the residents' quality of life. The international experience simultaneously showed that the socioeconomic benefits of preventive health care will be revealed after some time and these benefits will be far greater than those brought about by simple medical treatments. Therefore, we believe that the reform of medical insurance payment patterns will soon control the growth of medical expenses by adhering to the correct direction and methods. Finally, "benefiting people is the ultimate goal of the development of health"; therefore, the ultimate goal of the reform of medical insurance payment patterns should be also to benefit the people. Thus, the success or failure of medical insurance reform shall not be measured only by the effect of controlling costs. The relationship between medical treatment and medical insurance should facilitate the role of medical insurance as economic leverage. Accordingly, the medical service providers form positive incentives and appropriate medical orientations, while patients demanding health care may form good habits of seeking medical treatment and healthy life, but not pursuing economic benefits through the medical insurance reform.

\section{Abbreviations}

WHO: the World Health Organization

\section{Declarations}

\section{Acknowledgements}

The project has received support from many parties, including Luohu District Government, Shenzhen Health and Health Committee, Shenzhen Human Resources and Social Security Bureau, and Shenzhen Medical Information Center. The Luohu District Government has given financial support. Through field visits and research, Shenzhen Human Resources and Social Security Bureau and Shenzhen Health and Health Committee have determined that Luohu Hospital Group will be the only pilot unit to explore the establishment of health-oriented reform of medical insurance payment methods and support the reform effect of the project team. to evaluate. The Shenzhen Medical Information Center provides information on the medical treatment information and medical insurance fund expenditures of the insured persons who have signed contracts with the hospital group.

\section{Authors' contributions}

Page $11 / 18$ 
All authors contributed to write this essay, Fangfang Gong is responsible for the article writing,field trips and data analysis. Xizhuo Sun, the dean of the Luohu hospital is in charge of the project maangement, review and revision of the article content, also finalization. While Wenhai Li goes to the field trips,dagta collection and statstics. Zou Zhang invests on-the-spot,does research on the reform of payment methods for medical insurance in China for 10 years,and studies relevant national policies, then finally Yanan Li translate this article to English.

\section{Funding}

This project has no funding.

\section{Availability of data and materials}

Participants did not provide consent to share their data beyond the original study team. The study team would be happy to interrogate the data on behalf of others upon reasonable request to the corresponding author.

\section{Ethics approval and consent to participate}

This article does not contain any studies with human participants or animals performed by any of the authors.

\section{Consent for publication}

Not applicable.

\section{Competing interests}

The authors declare that they have no competing interests.

\section{References}

1 Pengqi Fang. China Medical and Health Care Development Report. People's Publishing House, Beijing,2016.

2 Ten years of medical reform and development: the reform of "three medical linkages" should be included in the healthy China strategy. 2019.https://baijiahao.baidu.com/s?id = $1629440477659545449 \& \mathrm{wfr}=$ spider\&for $=$ pc.html. $($ accessed March 30, 2019).

3 National Health and Health Commission: Resolving the problem of poverty caused by illness and returning to poverty, winning the battle against poverty.2018.http://health.people.com.cn/n1/2018/0425/c14739-29949739.html. 『accessed April 25, 2018). 
4 Pengqi Fang.China Medical and Health Care Development Report. People's Publishing House, Beijing,2016.

5 Kingson E R, Cornman JM. Health Care Reform: Universal Aces Is Feasible and Necessary. Benefits Quarterly Third Quarter, 2017;23(3):27-34

6 A. B. Flood et al. "How Do HMOs Achieve Savings? The Effectiveness of One Organization's Strategies". Health Services Research. 1998;33(1):79-99

7 Fangfang Gong, Xizhuo Sun, Wei Liu. The phased effect of Luohu medical insurance payment method reform. Chinese hospital,2017;21(11):7-9

8 Miao Yudong. Medical-medical insurance collaborative management model and strategy research [D]. Wuhan: Huazhong University of Science and Technology,2017.

\section{Tables}

Table 1: Total health expenditure structure in China

\begin{tabular}{|c|c|c|c|c|c|c|}
\hline \multirow[t]{2}{*}{ Year } & \multicolumn{2}{|c|}{ Government expenditure on health } & \multicolumn{2}{|l|}{ Social health expenditure } & \multicolumn{2}{|l|}{ Personal health expenditure } \\
\hline & Amount (100 million yuan) & Proportion (\%) & Amount (100 million yuan) & Proportion (\%) & Amount (100 million yuan) & Proportion (\%) \\
\hline 2009 & $4,816.26$ & 27.46 & $6,154.49$ & 35.08 & $6,571.16$ & 37.46 \\
\hline 2010 & $5,732.49$ & 28.69 & $7,196.61$ & 36.02 & $7,051.29$ & 35.29 \\
\hline 2011 & $7,464.18$ & 30.66 & $8,416.45$ & 34.57 & $8,465.28$ & 34.77 \\
\hline 2012 & $8,431.98$ & 29.99 & $10,030.70$ & 35.67 & $9,556.32$ & 34.34 \\
\hline 2013 & $9,545.81$ & 30.14 & $11,393.79$ & 35.98 & $10,729.34$ & 33.88 \\
\hline 2014 & $10,579.23$ & 29.96 & $13,437.75$ & 38.05 & $11,295.41$ & 31.99 \\
\hline 2015 & $12,475.28$ & 30.45 & 16506.71 & 40.29 & $11,992.65$ & 29.27 \\
\hline 2016 & $13,910.31$ & 30.01 & $19,096.68$ & 41.21 & $13,337.90$ & 28.78 \\
\hline 2017 & $15,205.87$ & 28.91 & $22,258.81$ & 42.32 & $15,133.60$ & 28.77 \\
\hline 2018 & $16,390.7$ & 28.3 & $24,944.7$ & 43 & $16,662.9$ & 28.7 \\
\hline
\end{tabular}

Data source: China Statistical Yearbook 2018

Table 2: Changes in the expenditure of per capita hospitalization medical insurance fund of the contracted insured 


\begin{tabular}{llll}
\hline Year & $\begin{array}{l}\text { Expenditure of per capita hospitalization medical } \\
\text { insurance fund (yuan) }\end{array}$ & $\begin{array}{l}\text { Annual } \\
\text { growth rate }\end{array}$ & $\begin{array}{l}\text { Annual growth rate of the expenditure of per capita medical } \\
\text { insurance fund in Shenzhen }\end{array}$ \\
\hline 2016 & 1,477 & - & - \\
2017 & 1,537 & $4.06 \%$ & $13 \%$ \\
2018 & 1,689 & $9.88 \%$ & $11 \%$ \\
\hline
\end{tabular}

Table 3: Changes in the top five disease types of the number of hospitalizations of the contracted insured from 2016 to 2018

\begin{tabular}{llll}
\hline Ranking & In 2016 & In 2017 & In 2018 \\
\hline 1 & Diseases like cervicalgia, omalgia, & Diseases like cervicalgia, omalgia, & Diseases like cervicalgia, omalgia, \\
& osphyalgia, and skelalgia & osphyalgia, and skelalgia & osphyalgia, and skelalgia \\
2 & Diseases like pulmonary infection & Diseases like pulmonary infection & Postoperative chemotherapy for malignant \\
& & & tumors \\
3 & Postoperative chemotherapy for malignant & Postoperative chemotherapy for malignant & Diseases like pulmonary infection \\
\hline 4 & tumors & tumors & Type 2 diabetes \\
\hline
\end{tabular}

\section{Figures}

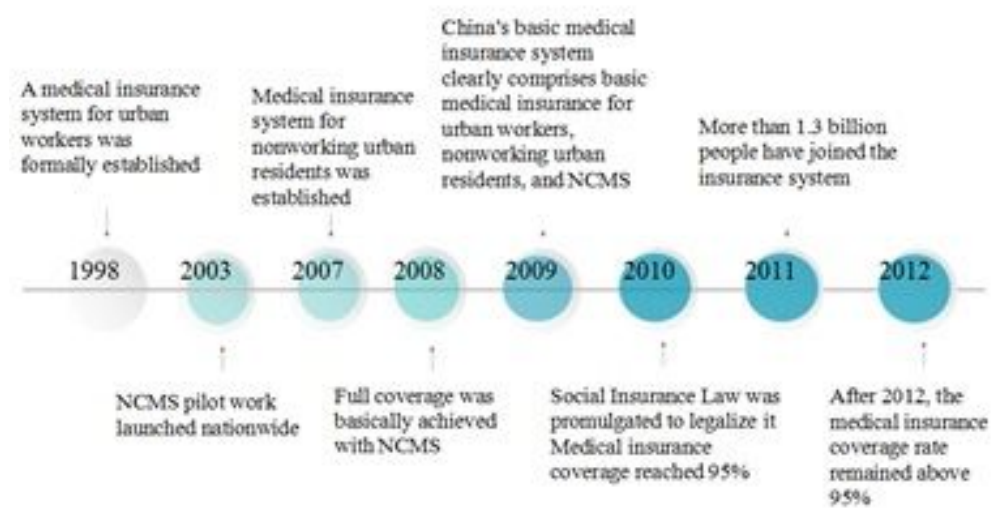

\section{Figure 1}

Progress chart for promoting universal medical Insurance in China 


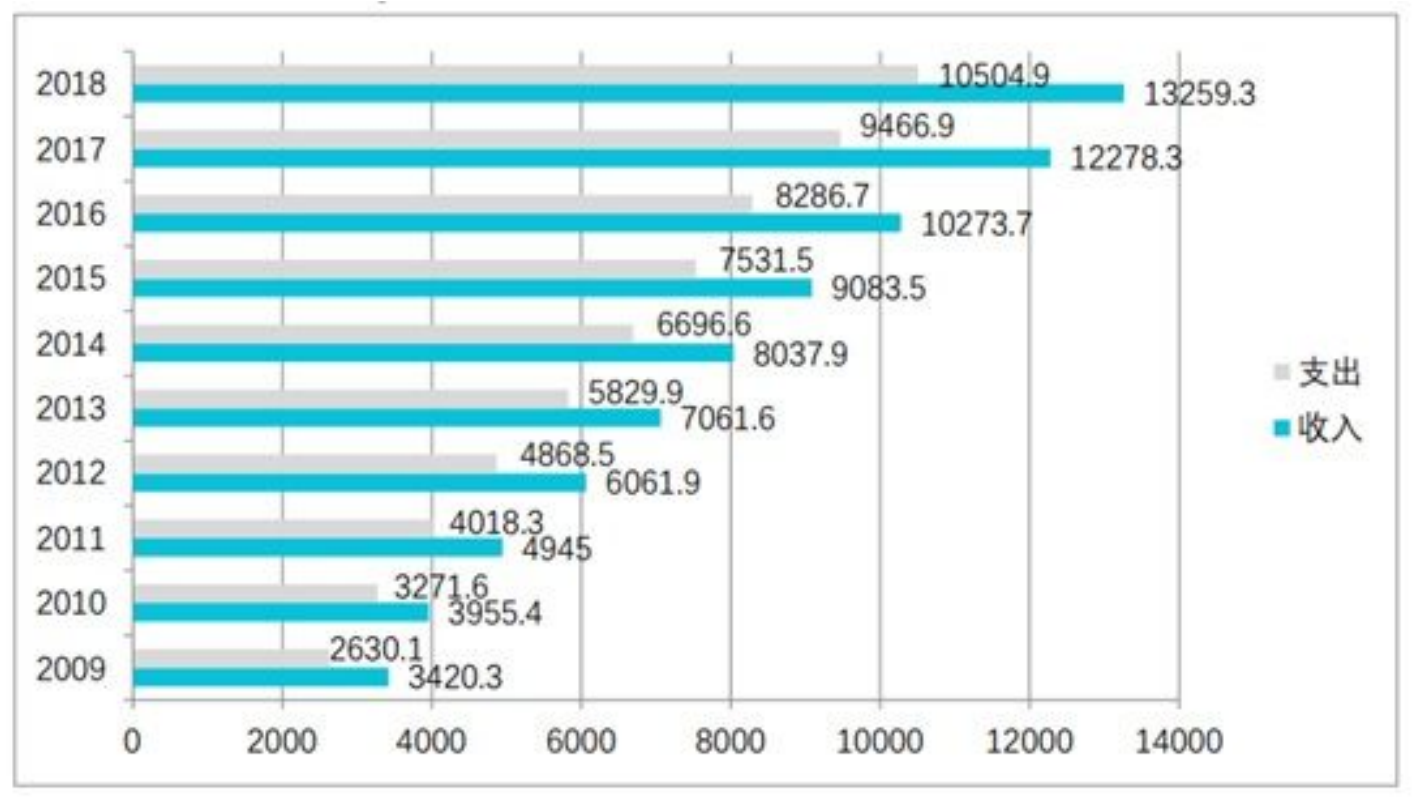

\section{Figure 2}

Income and expenditure changes in employee medical insurance funds from 2009 to 2018

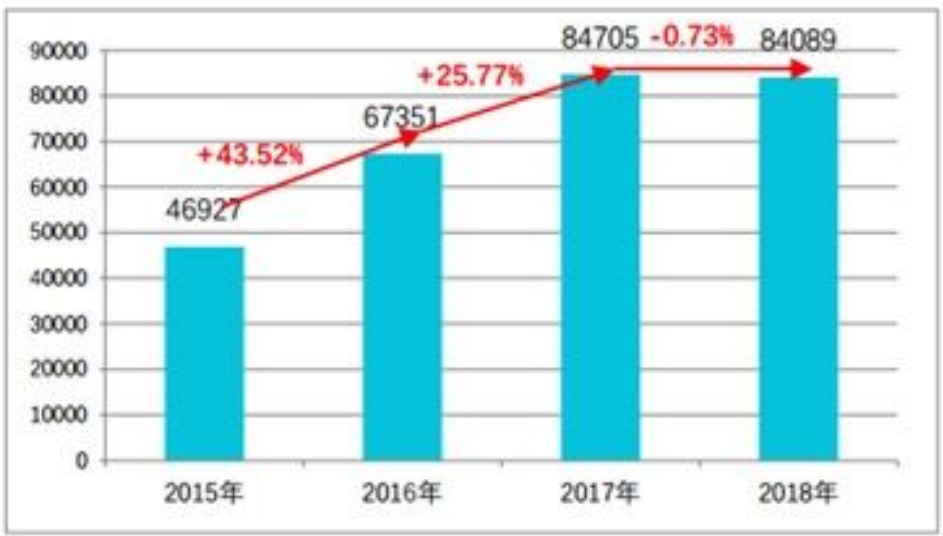

Figure 3

Changes in the total cost of hospitalization for the contracted insured (10,000 yuan) 


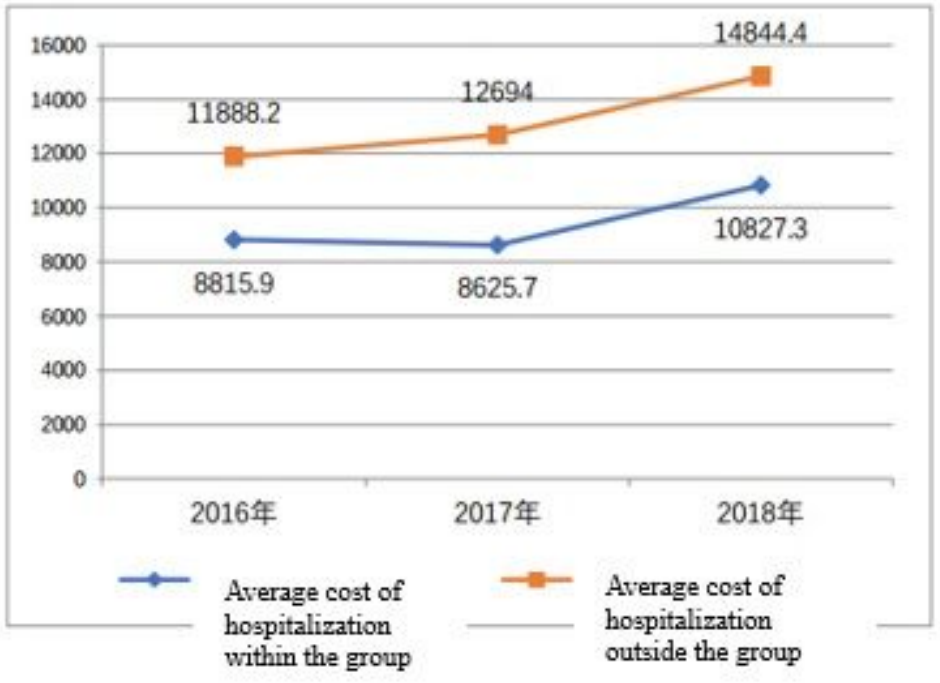

Figure 4

Changes in the average cost of hospitalizations for the contracted insured (yuan)

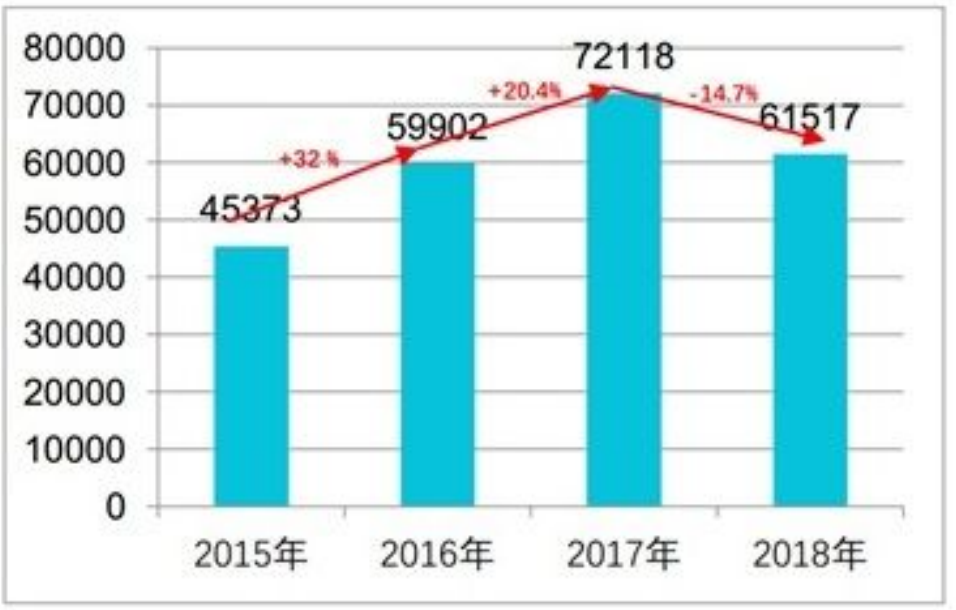

Figure 5

Changes in the number of hospitalizations for the contracted insured

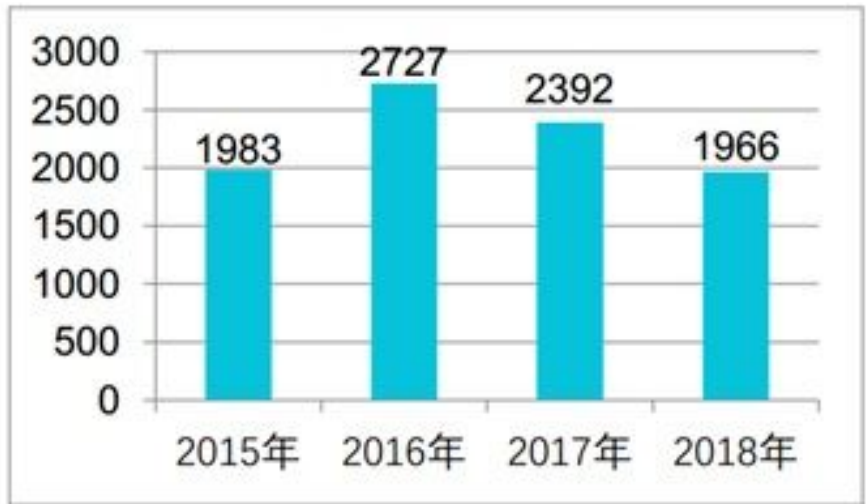

Figure 6 
Changes in the number of hospitalizations for diseases, such as pulmonary infections (case)

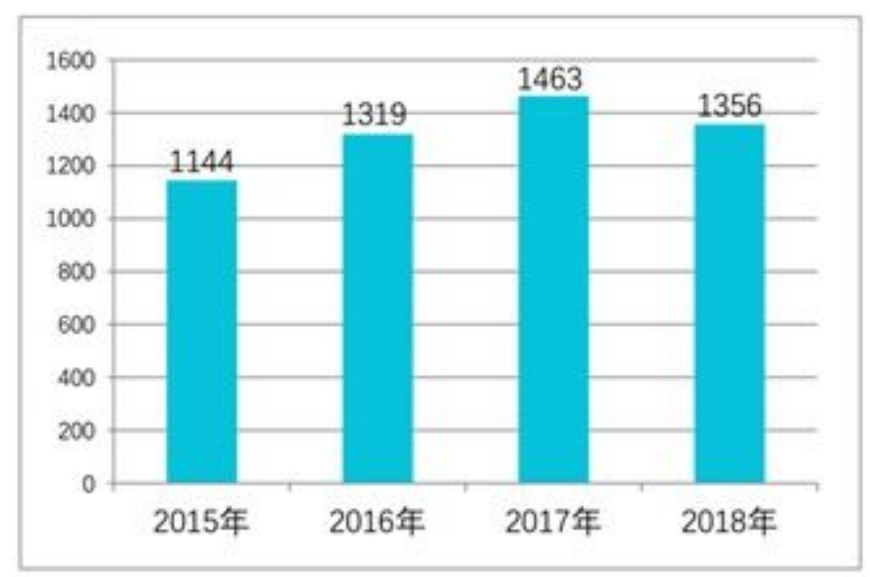

\section{Figure 7}

Changes in the number of hospitalizations for type 2 diabetes

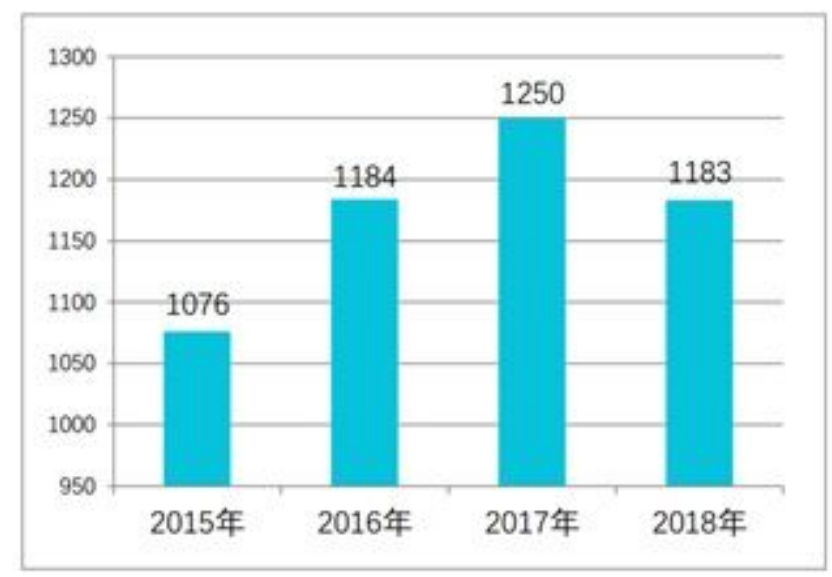

\section{Figure 8}

Changes in the number of hospitalizations for hypertension

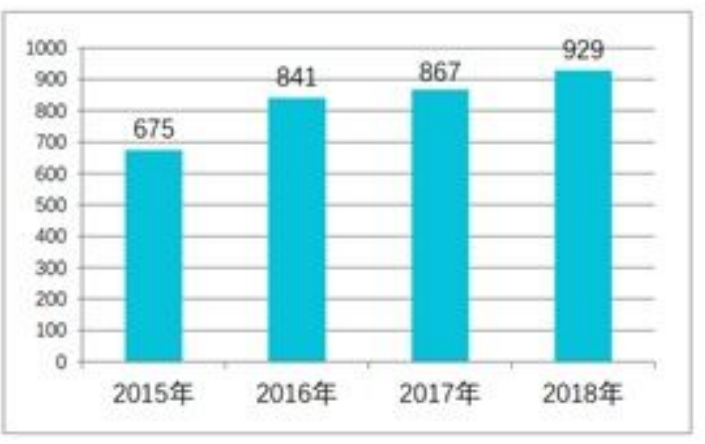

\section{Figure 9}

Changes in the number of hospitalizations for senile cataract 


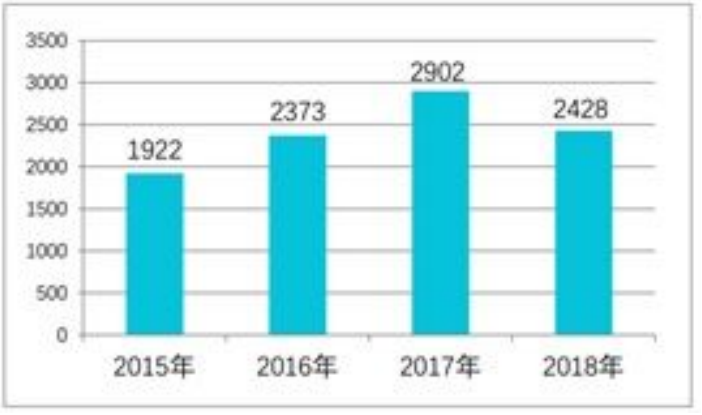

\section{Figure 10}

Changes in the number of hospitalizations for postoperative chemotherapy of malignant tumors

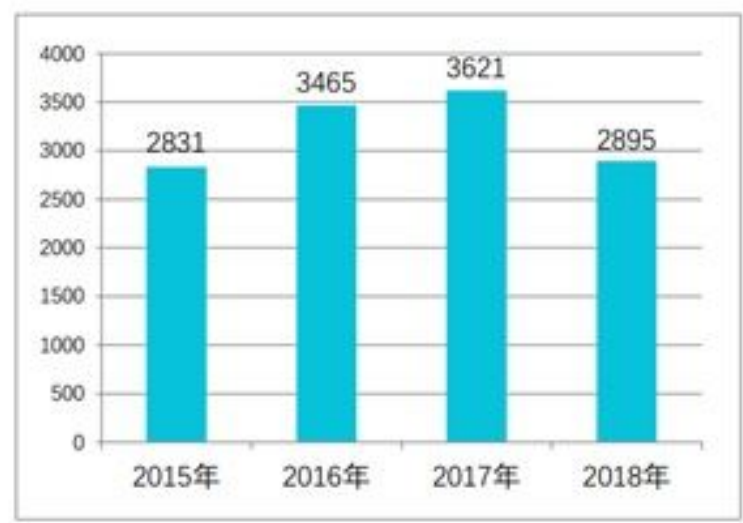

\section{Figure 11}

Changes in the number of hospitalizations for diseases such as cervicalgia, omalgia, osphyalgia, and skelalgia 